\title{
FAITHFUL ACTIONS OF LOCALLY COMPACT QUANTUM GROUPS ON CLASSICAL SPACES
}

\author{
DEBASHISH GOSWAMI AND SUTANU ROY
}

\begin{abstract}
It is well-known that no non-Kac compact quantum group can faithfully act on $\mathrm{C}(X)$ for a classical, compact Hausdorff space $X$. However, in this article we show that this is no longer true if we go to non-compact spaces and non-compact quantum groups, by exhibiting a large class of examples of locally compact quantum groups coming from bicrossed product construction, including non-Kac ones, which can faithfully and ergodically act on classical (non-compact) spaces. However, none of these actions can be isometric in the sense of [5], leading to the conjecture that the result obtained by Goswami and Joardar in 6] about non-existence of genuine quantum isometry of classical compact connected Riemannian manifolds may hold in the non-compact case as well.
\end{abstract}

\section{INTRODUCTION}

Symmetry plays a crucial role in many areas of mathematics and physics. Conventionally, group actions are used to model symmetries and with the advent of more general mathematical structures called quantum groups in [3,9,17. It should be natural to consider the actions (defined in a suitable sense depending on the algebraic or analytic framework chosen) of quantum groups on classical and noncommutative spaces. In this context, a very interesting programme is to study quantum symmetries of classical spaces. One may hope that there are many more quantum symmetries of a given classical space than classical group symmetries which will help one understand the space better. Indeed, it has been a remarkable discovery of S. Wang that for $n \geq 4$, a finite set of cardinality $n$ has an infinite dimensional compact quantum group ('quantum permutation group') of symmetries. For the relevance of quantum group symmetries in a wider and more geometric context, we refer the reader to the discussion on 'hidden symmetry in algebraic geometry' in [12, Chapter 13] where Manin made a remark about possible genuine Hopf algebra symmetries of classical smooth algebraic varieties.

Recently, several examples of faithful continuous action by genuine (i.e. not of the form $\mathrm{C}(G)$ for a compact group $G)$ compact quantum groups on $\mathrm{C}(X)$ for a connected compact space $X$ were constructed by H. Huang [7]. In [4 an example of a faithful action by the finite dimensional genuine compact quantum group on the algebra of regular function of a non-smooth variety was given. However, it turns out rather formidable to construct such actions when the space is smooth (and connected) and the action is also smooth in some natural sense. In 6], it is conjectured that no smooth faithful action of genuine compact quantum group on a compact connected smooth manifold can exist. The conjecture has been proved in

2010 Mathematics Subject Classification. 81R50, 46L89.

Key words and phrases. Bicrossed product, faithful $\mathrm{C}^{*}$-action, locally compact quantum group.

The first author was partially supported by Swarnajayanti grant given by D.S.T., Government of India. The second author was supported by the visiting scientist program at the Indian Statistical Institute, Kolkata. Parts of the manuscript had been written and revised while the second author had been supported by Fields-Ontario postdoctoral fellowship, NSERC and ERA at the University of Ottawa and Carleton University. 
that paper in two important cases: (i) when the action is isometric and (ii) when the compact quantum group is finite dimensional. We also mention the work of Etingof and Walton [4] which gives a similar no go result in the algebraic framework.

It is, however, expected that genuine non-compact quantum groups may have faithful smooth actions on smooth connected manifolds. Indeed, there are examples of such actions in the algebraic set-up (see [6, Example 14.2]). This motivates one to construct examples of faithful $\mathrm{C}^{*}$-actions of non-compact locally compact quantum groups on $\mathrm{C}_{0}(X)$ where $X$ is a smooth connected manifold. It is also desirable to see if the actions are smooth in any suitable sense. We give a method to construct such actions using the theory of bicrossed products due to Baaj, Skandalis and Vaes [1] and Vaes and Vainermann [15]. There is one remarkable observation: there are several non-Kac quantum groups with faithful $\mathrm{C}^{*}$-actions (even ergodic) on $\mathrm{C}_{0}(X)$. This could not be possible in the realm of compact quantum groups, as any compact quantum group acting faithfully on a commutative $\mathrm{C}^{*}$ - algebra must be of Kac type (see [8]). Thus, there seems to be more freedom for getting quantum symmetries on classical spaces in the realm of locally compact quantum groups than the compact ones. On the other hand, it should be noted that if we are interested in actions which are isometric in a natural sense (as in [5]). We cannot possibly get any genuine locally compact quantum group actions on classical (connected) Riemannian manifolds. Indeed, such a no-go result is obtained within the class of locally compact quantum groups considered by us in this paper (see Theorem 4.7).

The plan of the paper is as follows. We gather some basic definitions and facts about locally compact quantum groups and their actions in the von Neumann as well as $\mathrm{C}^{*}$-algebraic set-up in Subsection 2.1. followed by a brief account of the bicrossed product construction of locally compact groups in Subsection 2.2. In Section [3, we specialise to the locally compact quantum groups arising from bicrossed product construction of two groups say $G_{1}, G_{2}$ forming a matched pair, with $G_{1}$ being an abelian Lie group. We describe a natural action of this bicrossed product quantum group on $\mathrm{C}_{0}\left(\widehat{G_{1}}\right)$ and verify that it satisfies Podleś-type density conditions. Section 4 is devoted to investigate necessary and sufficient conditions for this action to be faithful or isometric. Using this, we observe that that there is a large class of genuine locally compact quantum groups having faithful actions on commutative $\mathrm{C}^{*}$-algebra of $\mathrm{C}_{0}$-functions on a locally compact manifold in Section 5 . However, it is shown in Subsection 4.2 that no such genuine quantum group actions can be isometric.

\section{Preliminaries}

All Hilbert spaces and $\mathrm{C}^{*}$-algebras are assumed to be separable.

For two norm-closed subsets $X$ and $Y$ of a $\mathrm{C}^{*}$-algebra, let

$$
X \cdot Y:=\{x y: x \in X, y \in Y\}^{\mathrm{CLS}},
$$

where CLS stands for the closed linear span.

For a $\mathrm{C}^{*}$-algebra $A$, let $\mathcal{M}(A)$ be its multiplier algebra and $\mathcal{U}(A)$ be the group of unitary multipliers of $A$. The unit of $\mathcal{M}(A)$ is denoted by $1_{A}$. Next recall some standard facts about multipliers and morphisms of $\mathrm{C}^{*}$-algebras from [13, Appendix $\mathrm{A}]$. Let $A$ and $B$ be $\mathrm{C}^{*}$-algebras. A *-homomorphism $\varphi: A \rightarrow \mathcal{M}(B)$ is called nondegenerate if $\varphi(A) \cdot B=B$. Each nondegenerate ${ }^{*}$-homomorphism $\varphi: A \rightarrow$ $\mathcal{M}(B)$ extends uniquely to a unital ${ }^{*}$-homomorphism $\widetilde{\varphi}$ from $\mathcal{M}(A)$ to $\mathcal{M}(B)$. Let $\mathfrak{C}^{*} \mathfrak{a l g}$ be the category of $\mathrm{C}^{*}$-algebras with nondegenerate ${ }^{*}$-homomorphisms $A \rightarrow$ $\mathcal{M}(B)$ as morphisms $A \rightarrow B$; let $\operatorname{Mor}(\mathrm{A}, \mathrm{B})$ denote this set of morphisms. We use the same symbol for an element of $\operatorname{Mor}(A, B)$ and its unique extension from $\mathcal{M}(A)$ to $\mathcal{M}(B)$. 
A representation of a $\mathrm{C}^{*}$-algebra $A$ on a Hilbert space $\mathcal{H}$ is a nondegenerate *-homomorphism $\pi: A \rightarrow \mathbb{B}(\mathcal{H})$. Since $\mathbb{B}(\mathcal{H})=\mathcal{M}(\mathbb{K}(\mathcal{H}))$, the nondegeneracy conditions $\pi(A) \cdot \mathbb{K}(\mathcal{H})=\mathbb{K}(\mathcal{H})$ is equivalent to begin $\pi(A)(\mathcal{H})$ is norm dense in $\mathcal{H}$, and hence this is same as having a morphism from $A$ to $\mathbb{K}(\mathcal{H})$. The identity representation of $\mathbb{K}(\mathcal{H})$ on $\mathcal{H}$ is denoted by $\operatorname{id}_{\mathcal{H}}$. The group of unitary operators on a Hilbert space $\mathcal{H}$ is denoted by $\mathcal{U}(\mathcal{H})$. The identity element in $\mathcal{U}(\mathcal{H})$ is denoted by $1_{\mathcal{H}}$.

We use $\otimes$ both for the tensor product of Hilbert spaces, minimal tensor product of $\mathrm{C}^{*}$-algebras, and von Neumann algebras which is well understood from the context. We write $\Sigma$ for the tensor flip $\mathcal{H} \otimes \mathcal{K} \rightarrow \mathcal{K} \otimes \mathcal{H}, x \otimes y \mapsto y \otimes x$, for two Hilbert spaces $\mathcal{H}$ and $\mathcal{K}$. We write $\sigma$ for the tensor flip isomorphism $A \otimes B \rightarrow B \otimes A$ for two $\mathrm{C}^{*}$-algebras or von Neumann algebras $A$ and $B$.

Let $A_{1}, A_{2}, A_{3}$ be $\mathrm{C}^{*}$-algebras. For any $t \in \mathcal{M}\left(A_{1} \otimes A_{2}\right)$ we denote the leg numberings on the level of $\mathrm{C}^{*}$-algebras as $t_{12}:=t \otimes 1_{A_{3}} \in \mathcal{M}\left(A_{1} \otimes A_{2} \otimes A_{3}\right), t_{23}:=$ $1_{A_{3}} \otimes t_{12} \in \mathcal{M}\left(A_{3} \otimes A_{1} \otimes A_{2}\right)$ and $t_{13}:=\sigma_{12}\left(t_{23}\right)=\sigma_{23}\left(t_{12}\right) \in \mathcal{M}\left(A_{1} \otimes A_{3} \otimes A_{2}\right)$. In particular, let $A_{i}=\mathbb{B}\left(\mathcal{H}_{i}\right)$ for some Hilbert spaces $\mathcal{H}_{i}$, where $i=1,2,3$. Then for any $t \in \mathbb{B}\left(\mathcal{H}_{1} \otimes \mathcal{H}_{2}\right)$ the leg numberings are obtained by replacing $\sigma$ with the conjugation by $\Sigma$ operator.

2.1. Locally compact quantum groups and their actions. For a general theory of $\mathrm{C}^{*}$-algebraic locally compact quantum groups we refer [11,13].

A C ${ }^{*}$-bialgebra $\left(A, \Delta_{A}\right)$ is a $\mathrm{C}^{*}$-algebra $A$ and a comultiplication $\Delta_{A} \in \operatorname{Mor}(A, A \otimes$ $A)$ that is coassociative: $\left(\operatorname{id}_{A} \otimes \Delta_{A}\right) \circ \Delta_{A}=\left(\Delta_{A} \otimes \operatorname{id}_{A}\right) \circ \Delta_{A}$. Moreover, $\left(A, \Delta_{A}\right)$ is bisimplifiable $\mathrm{C}^{*}$-bialgebra if $\Delta_{A}$ satisfies the cancellation property

$$
\Delta_{A}(A) \cdot\left(1_{A} \otimes A\right)=\Delta_{A}(A) \cdot\left(A \otimes 1_{A}\right)=A \otimes A .
$$

Let $\varphi$ be a faithful (approximate) KMS weight on $A$ (see [10, Section 1]). The set of all positive $\varphi$-integrable and $\varphi$-square integrable elements are defined by $\mathcal{M}_{\varphi}^{+}:=$ $\left\{a \in A^{+} \mid \varphi(a)<\infty\right\}$ and $\mathcal{N}_{\varphi}:=\left\{a \in A \mid \varphi\left(a^{*} a\right)<\infty\right\}$, respectively. Moreover, $\varphi$ is called

(1) left invariant if $\omega\left(\left(\operatorname{id}_{A} \otimes \varphi\right) \Delta_{A}(a)\right)=\omega(1) \varphi(a)$ for all $\omega \in A_{*}^{+}, a \in \mathcal{M}_{\varphi}^{+}$;

(2) right invariant if $\omega\left(\left(\varphi \otimes \operatorname{id}_{A}\right) \Delta_{A}(a)\right)=\omega(1) \varphi(a)$ for all $\omega \in A_{*}^{+}, a \in \mathcal{M}_{\varphi}^{+}$.

Definition 2.2 ([10, Definition 4.1]). A locally compact quantum group (quantum groups from now onwards) is a bisimplifiable $\mathrm{C}^{*}$-bialgebra $\mathbb{G}=\left(A, \Delta_{A}\right)$ with left and right invariant approximate $\mathrm{KMS}$ weights $\varphi$ and $\psi$, respectively.

By [10, Theorem $7.14 \& 7.15]$, invariant weights $\varphi$ and $\psi$ are unique up to a positive scalar factor; hence they are called the left and right Haar weights for $\mathbb{G}$. Moreover, there is a unique (up to isomorphism) Pontrjagin dual $\widehat{\mathbb{G}}=\left(\hat{A}, \hat{\Delta}_{A}\right)$ of $\mathbb{G}$, which is again a quantum group.

Next we consider the GNS triple $\left(\mathrm{L}^{2}(\mathbb{G}), \pi, \Lambda\right)$ for $\varphi$. There is an element $\mathbb{W} \in$ $\mathcal{U}\left(\mathrm{L}^{2}(\mathbb{G}) \otimes \mathrm{L}^{2}(\mathbb{G})\right)$ satisfying the pentagon equation:

$$
\mathbb{W}_{23} \mathbb{W}_{12}=\mathbb{W}_{12} \mathbb{W}_{13} \mathbb{W}_{23} \quad \text { in } \mathcal{U}\left(\mathrm{L}^{2}(\mathbb{G}) \otimes \mathrm{L}^{2}(\mathbb{G}) \otimes \mathrm{L}^{2}(\mathbb{G})\right)
$$

$\mathbb{W}$ is called multiplicative unitary. Furthermore, [10, Proposition 6.10] shows that $\mathbb{W}$ is manageable (in the sense of $[18$, Definition 1.2]). Also $\mathbb{W}$ generates $\mathbb{G}$ (or $\mathbb{G}$ is generated by $\mathbb{W}$ ) in the following sense:

(1) the dual multiplicative unitary $\widehat{\mathbb{W}}:=\Sigma \mathbb{W}^{*} \Sigma \in \mathcal{U}\left(\mathrm{L}^{2}(\mathbb{G}) \otimes \mathrm{L}^{2}(\mathbb{G})\right)$ is also manageable.

(2) the slices of $\mathbb{W}$ defined by

$$
\begin{aligned}
& \hat{A}:=\left\{\left(\omega \otimes \mathrm{id}_{\mathrm{L}^{2}(\mathbb{G})}\right) \mathbb{W}: \omega \in \mathbb{B}\left(\mathrm{L}^{2}(\mathbb{G})\right)_{*}\right\}^{\mathrm{CLS}}, \\
& A:=\left\{\left(\mathrm{id}_{\mathrm{L}^{2}(\mathbb{G})} \otimes \omega\right) \mathbb{W}: \omega \in \mathbb{B}\left(\mathrm{L}^{2}(\mathbb{G})\right)_{*}\right\}^{\mathrm{CLS}},
\end{aligned}
$$


are nondegenerate $\mathrm{C}^{*}$-subalgebras of $\mathbb{B}\left(\mathrm{L}^{2}(\mathbb{G})\right)$.

(3) $\mathbb{W} \in \mathcal{U}(A \otimes \hat{A}) \subseteq \mathcal{U}\left(\mathrm{L}^{2}(\mathbb{G}) \otimes \mathrm{L}^{2}(\mathbb{G})\right)$.

(4) the comultiplication maps $\Delta_{A}$ and $\hat{\Delta}_{A}$ are defined by

$$
\Delta_{A}(a):=\mathbb{W}^{*}(1 \otimes a) \mathbb{W}, \quad \hat{\Delta}_{A}(\hat{a}):=\sigma\left(\mathbb{W}(\hat{a} \otimes 1) \mathbb{W}^{*}\right),
$$

for all $a \in A, \hat{a} \in \hat{A}$.

A general theory of locally compact quantum groups in the von-Neumann algebraic framework has been developed by Kustermans and Vaes in [11. Moreover, there is a nice interplay between $\mathrm{C}^{*}$-algebraic and von-Neumann algebraic locally compact quantum groups in general via multiplicative unitaries. We briefly recall this in the group case.

Let $G$ be a locally compact group and $\mu$ be its left Haar measure. Define, $\mathbb{W} \in$ $\mathcal{U}\left(L^{2}(G \times G, \mu \times \mu)\right.$ by $\mathbb{W} \xi(x, y)=\xi\left(x, x^{-1} y\right)$ for all $\xi \in L^{2}(G \times G, \mu \times \mu), x, y \in G$. A simple computation shows that $\mathbb{W}$ is a multiplicative unitary. Furthermore,

$$
\begin{aligned}
A & =\mathrm{C}_{0}(G)=\left\{\left(\operatorname{id}_{\mathrm{L}^{2}(G, \mu)} \otimes \omega\right) \mathbb{W}: \omega \in \mathbb{B}\left(\mathrm{L}^{2}(G)\right)_{*}\right\}^{\mathrm{CLS}}, \\
M & =L^{\infty}(G)=\left\{\left(\operatorname{id}_{\mathrm{L}^{2}(\mathbb{G})} \otimes \omega\right) \mathbb{W}: \omega \in \mathbb{B}\left(\mathrm{L}^{2}(\mathbb{G})\right)_{*}\right\}^{\text {weak closure }} .
\end{aligned}
$$

Using (2.6) we get $\Delta_{M}: L^{\infty}(G) \rightarrow L^{\infty}(G \times G)$ by $\Delta_{M}(f)(x, y)=f(x y)$ for all $f \in$ $L^{\infty}(G)$. The pair $\left(M, \Delta_{M}\right)$ is a von-Neumann bialgebra. The left Haar weight $\varphi$ on $L^{\infty}(G)$ is given by the integration with respect to the left Haar measure $\mu$ on $G: \varphi(f):=\int f(h) \mathrm{d} \mu(h)$ for $f \in L^{\infty}\left(G_{2}\right)^{+}$. Similarly, the right Haar weight $\psi$ on $\left(M, \Delta_{M}\right)$ is obtained from the to the right Haar measure on $G$. The pair $\mathbb{G}=$ $\left(M, \Delta_{M}\right)$ is the von Neumann algebraic locally compact quantum group associated to $G$.

Similarly, $\Delta_{A}: \mathrm{C}_{0}(G) \rightarrow \mathrm{C}_{\mathrm{b}}(G \times G)$ is obtained by restricting $\Delta_{M}$ on $\mathrm{C}_{0}(G)$. The restriction of left and right Haar weight of $\left(M, \Delta_{M}\right)$ on $\mathrm{C}_{0}(G)$ defines the left and Haar weight on $\left(A, \Delta_{A}\right)$, respectively. Thus $\left(A, \Delta_{A}\right)$ is the $\mathrm{C}^{*}$-algebraic version of $\mathbb{G}$.

Let $\hat{A}=\mathrm{C}_{\mathrm{r}}^{*}(G)$ and $\hat{M}=L(G)$. Let $\lambda$ be the left regular representation of $G$ on $L^{2}(G, \mu)$. Define, $\hat{\Delta}_{M}: L(G) \rightarrow L(G \times G)$ by $\hat{\Delta}\left(\lambda_{g}\right)=\lambda_{g} \otimes \lambda_{g}$ and $\hat{\Delta}_{A}: \mathrm{C}_{\mathrm{r}}^{*}(G) \rightarrow$ $\mathcal{M}\left(\mathrm{C}_{\mathrm{r}}^{*}(G \times G)\right)$ as the restriction of $\hat{\Delta}_{M}$. The left and right invariant invariant Haar weights on $\hat{M}$ is given by $\hat{\varphi}(\lambda(f))=f\left(1_{G}\right)$ for all $f \in \mathrm{C}_{\mathrm{c}}(G)$ such that $\lambda(f) \in L(G)^{+}$. Then $\widehat{\mathbb{G}}=\left(\hat{A}, \hat{\Delta}_{A}\right)$ and $\widehat{\mathbb{G}}=\left(\hat{M}, \hat{\Delta}_{M}\right)$ are the dual of $\mathbb{G}$ in $\mathrm{C}^{*}$-algebraic and von Neumann algebraic setting, respectively.

Definition 2.7. A (right) $\mathrm{C}^{*}$-action of $\mathbb{G}$ on a $\mathrm{C}^{*}$-algebra $C$ is a morphism $\gamma: C \rightarrow$ $C \otimes A$ with the following properties:

(1) $\gamma$ is a comodule structure, that is,

$$
\left(\operatorname{id}_{C} \otimes \Delta_{A}\right) \gamma=\left(\gamma \otimes \operatorname{id}_{A}\right) \gamma ;
$$

(2) $\gamma$ satisfies the Podleś condition:

$$
\gamma(C) \cdot\left(1_{C} \otimes A\right)=C \otimes A .
$$

Some authors demands $\gamma$ to be injective. Similarly, the von Neumann algebraic version $\left(M, \Delta_{M}\right)$ of $\mathbb{G}$ acts on von Neumann algebras as well.

Definition 2.10. A (right) von Neumann algebraic action of $\mathbb{G}$ on a von Neumann algebra $N$ is a faithful, normal, unital ${ }^{*}$-homomorphism $\gamma: N \rightarrow N \otimes M$ satisfying $\left(\operatorname{id}_{N} \otimes \Delta_{M}\right) \gamma=\left(\gamma \otimes \operatorname{id}_{M}\right) \gamma$. 
2.2. Bicrossed product of groups. Bicrossed product construction for mathced pair of locally compact quantum groups goes back to the work of Baaj and Vaes [2] and Vaes and Vainerman [15. In this article, we shall restrict out attention to the bicrossed product construction for locally compact groups.

Definition 2.11 ([15, Definition 4.7]). Let $G_{1}, G_{2}$ and $G$ be locally compact groups with fixed left Haar measures. The pair $\left(G_{1}, G_{2}\right)$ is called a matched pair if

(1) there exist a homomorphism $i: G_{1} \hookrightarrow G$ and an anti-homomorphism $j: G_{2} \hookrightarrow$ $G$ with closed images and homeomorphism onto these images;

(2) $\theta: G_{1} \times G_{2} \rightarrow G$ defined by $\theta((g, h))=i(g) j(h)$ is an homeomorphism onto an open subgroup $\Omega$ of $G$ having a complement of measure zero.

This allows to define almost everywhere and measurable left action $\left(\alpha_{g}\right)_{g \in G_{1}}$ of $G_{1}$ on $G_{2}$ and right action $\left(\beta_{h}\right)_{h \in G_{2}}$ on $G$, and satisfies $j\left(\alpha_{g}(h)\right) i\left(\beta_{h}(g)\right)=$ $i(g) j(h)$ for almost all $g \in G_{1}$ and $h \in G_{2}$.

By [15, Lemma 4.9], the maps $G_{1} \times G_{2} \rightarrow G_{2}:(g, h) \mapsto \alpha_{g}(h)$ and $G_{1} \times G_{2} \rightarrow$ $G_{1}:(g, h) \mapsto \beta_{h}(g)$ are measurable, defined almost everywhere, and satisfy the following relations:

$$
\begin{aligned}
\alpha_{g s}(h) & =\alpha_{g}\left(\alpha_{s}(h)\right), & \beta_{h}(g s) & =\beta_{\alpha_{s}(h)}(g) \beta_{h}(s), \\
\beta_{h t}(g) & =\beta_{h}\left(\beta_{t}(g)\right), & \alpha_{g}(h t) & =\alpha_{\beta_{t}(g)}(h) \alpha_{g}(t) .
\end{aligned}
$$

for almost all $g, s \in G_{1}, h, t \in G_{2}$. Also, $\alpha_{g}\left(1_{G_{2}}\right)=1_{G_{2}}$, and $\beta_{h}\left(1_{G_{1}}\right)=1_{G_{1}}$ for all $g \in G, h \in H$.

Then $\alpha: L^{\infty}\left(G_{2}\right) \rightarrow L^{\infty}\left(G_{1} \times G_{2}\right)$ defined by $\alpha(f)(g, s):=f\left(\alpha_{g}(s)\right)$ is a (left) von Neumann algebraic action of the locally compact quantum group $\mathbb{G}_{1}=\left(\left(L^{\infty}\left(G_{1}\right), \Delta_{L^{\infty}\left(G_{1}\right)}\right)\right.$ on $L^{\infty}\left(G_{2}\right)$. The von Neumann algebraic version of the bicrossed product $\mathbb{G}=$ $\left(M, \Delta_{M}\right)$ is given by

$$
M:=\left(\alpha\left(L^{\infty}\left(G_{2}\right)\right)\left(L\left(G_{1}\right) \otimes 1\right)\right)^{\prime \prime}, \quad \Delta_{M}(z):=\mathbb{W}^{*}(1 \otimes z) \mathbb{W}
$$

Let $\lambda$ be the left regular representation of $G_{1}$. The left (and right) Haar weight on $L^{\infty}\left(G_{2}\right)$. Let $\hat{\alpha}$ be the dual action of $L\left(G_{1}\right)$ on the crossed product $M$. Then $\hat{\alpha}: M \rightarrow$ $L\left(G_{1}\right) \otimes M$ is defined by $\hat{\alpha}(\alpha(y))=1_{L\left(G_{1}\right)} \otimes \alpha(\eta)$ and $\hat{\alpha}\left(x \otimes 1_{L^{\infty}\left(G_{2}\right)}\right)=\hat{\Delta}_{L\left(G_{1}\right)}(x) \otimes$ $1_{L^{\infty}\left(G_{2}\right)}$ for all $x \in L\left(G_{1}\right)$ and $y \in L^{\infty}\left(G_{2}\right)$.

Let $\hat{\varphi}_{1}$ and $\varphi_{2}$ be the left Haar weights on $L\left(G_{1}\right)$ and $L^{\infty}\left(G_{2}\right)$, respectively. By [15. Definition 1.13], the left Haar weight $\varphi$ on $\mathbb{G}$ is given by $\varphi:=\varphi_{2} \alpha^{-1}\left(\hat{\varphi}_{1} \otimes\right.$ id $\otimes$ id) $\hat{\alpha}$. A simple computation shows, for any $f \in \mathcal{N}_{\hat{\varphi}_{1}}$ and $\eta \in \mathcal{N}_{\varphi_{2}}$,

$$
\varphi\left((\alpha(\eta)(\lambda(f) \otimes 1))=\hat{\varphi}_{1}(\lambda(f)) \varphi_{2}(\eta)=\varphi((\lambda(f) \otimes 1)(\alpha(\eta))\right.
$$

Let $\mathcal{H}=L^{2}\left(G_{1} \times G_{2}\right)$ be the Hilbert space of square integrable functions with respect to the product of the left Haar measures of $G_{1}$ and $G_{2}$. Using 1, Definition 3.3] for left Haar measure, we obtain a multiplicative unitary $\mathbb{W} \in \mathcal{U}(\mathcal{H} \otimes \mathcal{H})$ for $\mathbb{G}$ defined by

$$
\mathbb{W} \xi(g, s, h, t):=\xi\left(\beta_{\alpha_{g}(s)^{-1} t}(h) g, s, h, \alpha_{g}(s)^{-1} t\right),
$$

for $\xi \in L^{2}\left(G_{1} \times G_{2} \times G_{1} \times G_{2}\right)$, and for almost all $g, s \in G_{1}, h, t \in G_{2}$.

Finally, we recall the $\mathrm{C}^{*}$-algebraic version of $\mathbb{G}$ from [1, Section 3]. Equip the quotient space $G_{1} \backslash G$ with its canonical invariant measure class. Then embedding $G_{2} \rightarrow G_{1} \backslash G$ identifies $G_{2}$ with a Borel subset of $G_{1} \backslash G$ with complement of measure zero. Then [1, Proposition 3.2] gives an isomorphism between $L^{\infty}\left(G_{2}\right)$ and $L^{\infty}\left(G_{1} \backslash G\right)$; hence we can restrict $\alpha$ to $\mathrm{C}_{0}\left(G_{1} \backslash G\right)$. The $\mathrm{C}^{*}$-algebraic version $\left(A, \Delta_{A}\right)$ of $\mathbb{G}$ is given by [1, Proposition 3.6]

$$
A:=\alpha\left(\mathrm{C}_{0}\left(G_{1} \backslash G\right)\right) \cdot\left(\lambda\left(\mathrm{C}_{\mathrm{r}}^{*}\left(G_{1}\right)\right) \otimes 1\right), \quad \alpha \in \operatorname{Mor}\left(\mathrm{C}_{0}\left(G_{2}\right), A\right) .
$$

The dual $\widehat{\mathbb{G}}=\left(\hat{A}, \hat{\Delta}_{A}\right)$ is obtained by exchanging the roles of $G_{1}$ and $\alpha$ by $G_{2}$ and $\beta$, respectively. 


\section{Existence of $\mathrm{C}^{*}$-ACTIONS of BiCROssed PRODUCTS ON SPACES}

Let $G_{1}, G_{2}$ be locally compact groups and $\left(G_{1}, G_{2}\right)$ form a matched pairs. Let $\mathbb{G}=\left(A, \Delta_{A}\right)$ be the associated ( $\mathrm{C}^{*}$-algebraic) bicrossed product quantum group. From now onwards we assume that $G_{1}$ is abelian.

Theorem 3.1. There is a $\mathrm{C}^{*}$-action of $\mathbb{G}$ on $\mathrm{C}_{0}\left(\widehat{G_{1}}\right)$.

Throughout $\lambda$ denotes the (right) regular representation of $G_{1}: \lambda(f) \xi^{\prime}(h):=$ $\int f(g) \xi^{\prime}(h g) \mathrm{d} \mu_{1}(g)$, for $f \in \mathrm{C}_{\mathrm{c}}\left(G_{1}\right)$ and $\xi^{\prime} \in L^{2}\left(G_{1}\right)$.

Clearly, we have an element $i \in \operatorname{Mor}\left(\mathrm{C}_{\mathrm{r}}^{*}\left(G_{1}\right), A\right)$ and its extension, denoted by $i$ again, to $\mathcal{M}\left(\mathrm{C}_{\mathrm{r}}^{*}\left(G_{1}\right)\right)$ is given by $i(x):=x \otimes 1$ for all $x \in \mathcal{M}\left(\mathrm{C}_{\mathrm{r}}^{*}\left(G_{1}\right)\right)$. Hence, $\gamma:=\Delta_{A} \circ i$ is an element in $\operatorname{Mor}\left(\mathrm{C}_{\mathrm{r}}^{*}\left(G_{1}\right), A \otimes A\right)$. In order to interpret $\gamma$ as the desired $\mathrm{C}^{*}$-action in Theorem 3.1 following proposition will be crucial.

Proposition 3.2. $\gamma$ is $a^{*}$-homomorphism from $\mathrm{C}_{\mathrm{r}}^{*}\left(G_{1}\right)$ to $\mathcal{M}\left(\mathrm{C}_{\mathrm{r}}^{*}\left(G_{1}\right) \otimes A\right)$ and defined by

$$
\gamma(\lambda(f)) \xi(g, h, t):=\int f(z) \xi\left(g \beta_{\alpha_{h}(t)}(z), h z, t\right) \mathrm{d} \mu_{1}(z)
$$

for all $f \in \mathrm{C}_{\mathrm{c}}\left(G_{1}\right)$ and $\xi \in L^{2}\left(G_{1} \times G_{1} \times G_{2}\right)$.

Proof. The adjoint $\mathbb{W}^{*} \in \mathcal{U}\left(L^{2}\left(G_{1} \times G_{2} \times G_{1} \times G_{2}\right)\right)$ of the multiplicative unitary $\mathbb{W}$ of $\mathbb{G}$ in (2.16) is defined by

$$
\mathbb{W}^{*} \xi^{\prime}(g, s, h, t):=\xi^{\prime}\left(g^{\prime}, s, h, \alpha_{g^{\prime}}(s) t\right) \quad \text { for } \xi^{\prime} \in L^{2}\left(G_{1} \times G_{2} \times G_{1} \times G_{2}\right),
$$

where $g^{\prime}:=\beta_{t}(h)^{-1} g$ for all $g, h \in G_{1}$ and $s, t \in G_{2}$. Using the definition of the comultiplication 2.6 and the property (2.13) of $\beta$ we obtain

$$
\begin{aligned}
\gamma(\lambda(f)) \xi^{\prime}(g, s, h, t) & =\Delta_{A}\left(1_{L^{2}\left(G_{1} \times G_{2}\right)} \otimes \lambda(f) \otimes 1_{L^{2}\left(G_{2}\right)}\right) \xi^{\prime}(g, s, h, t) \\
& =\left(\mathbb{W}^{*}\left(1_{L^{2}\left(G_{1} \times G_{2}\right)} \otimes \lambda(f) \otimes 1_{L^{2}\left(G_{2}\right)}\right) \mathbb{W}\right) \xi^{\prime}(g, s, h, t) \\
& =\left(\left(1_{L^{2}\left(G_{1} \times G_{2}\right)} \otimes \lambda(f) \otimes 1_{L^{2}\left(G_{2}\right)}\right) \mathbb{W}\right) \xi^{\prime}\left(g^{\prime}, s, h, \alpha_{g^{\prime}}(s) t\right) \\
& =\mathbb{W}\left(\int f(z) \xi^{\prime}\left(g^{\prime}, s, h z, \alpha_{g^{\prime}}(s) t\right) \mathrm{d} \mu_{1}(z)\right) \\
& =\int f(z) \xi^{\prime}\left(g \beta_{\alpha_{h}(t)}(z), s, h z, t\right) \mathrm{d} \mu_{1}(z) .
\end{aligned}
$$

Since $G_{1}$ is abelian, we indenify $\mathcal{M}\left(\mathrm{C}_{\mathrm{r}}^{*}\left(G_{1}\right) \otimes A\right)$ with $\mathrm{C}_{\mathrm{b}}\left(\widehat{G_{1}}, \mathcal{M}(A)\right) \subset \mathbb{B}\left(L^{2}\left(\widehat{G_{1}} \times\right.\right.$ $\left.G_{1} \times G_{2}\right)$ ) using the Fourier transform.

Let $f: \widehat{G_{1}} \rightarrow \mathcal{M}(A)$ be a strictly continuous function. Define an operator $M_{f}$ acting on $L^{2}\left(\widehat{G_{1}} \times G_{1} \times G_{2}\right)$ by

$M_{f}\left(\xi_{1} \otimes \xi_{2}\right)(\hat{g}, h, t):=\xi_{1}(\hat{g})\left(f(\hat{g}) \xi_{2}\right)(h, t) \quad$ for all $\xi_{1} \in L^{2}\left(\widehat{G_{1}}\right), \xi_{2} \in L^{2}\left(G_{1} \times G_{2}\right)$, which is an element in $M_{f} \in \mathrm{C}_{\mathrm{b}}\left(\widehat{G_{1}}, \mathcal{M}(A)\right) \cong \mathcal{M}\left(\mathrm{C}_{\mathrm{r}}^{*}\left(G_{1}\right) \otimes A\right)$.

Recall the dual pairing $\langle\cdot, \cdot\rangle: G_{1} \times \widehat{G_{1}} \rightarrow \mathbb{T}$ defined by $\langle h, \hat{g}\rangle:=\hat{g}(h)$ for all $h \in G_{1}$ and $\hat{g} \in \widehat{G_{1}}$. Let $D$ be a $C^{*}$-algebra. For an element $F \in L^{1}\left(G_{1}, D\right)$, the Fourier transform $\widehat{F}$ is defined by $\widehat{F}(\hat{g}):=\int F(h)\langle h, \hat{g}\rangle \mathrm{d} \mu_{1}(h)$ for $\hat{g} \in \widehat{G_{1}}$, and $\widehat{F}$ is an element in $\mathrm{C}_{0}\left(\widehat{G_{1}}, D\right)$.

Then, for a fixed $z \in G_{1}$, consider $T_{z}: \widehat{G_{1}} \rightarrow \mathcal{M}(A)$ defined by

$$
T_{z}(\hat{g}) \xi_{2}(h, t):=\left\langle\beta_{\alpha_{h}(t)}(z), \hat{g}\right\rangle \xi_{2}(h z, t) \quad \text { for all } \xi_{2} \in L^{2}\left(G_{1} \times G_{2}\right) .
$$

Clearly, $\hat{g} \mapsto T_{z}(\hat{g})$ is continuous in the strict topology. Hence, any $f \in \mathrm{C}_{\mathrm{c}}\left(G_{1}\right)$ gives $\int f(z) T_{z} \mathrm{~d} \mu_{1}(z)$ is an element in $\mathrm{C}_{\mathrm{b}}\left(\widehat{G_{1}}, \mathcal{M}(A)\right) \cong \mathcal{M}\left(\mathrm{C}_{\mathrm{r}}^{*}\left(G_{1}\right) \otimes A\right)$. 
Using the Fourier transform in the first leg of (3.4) we observe

$$
\gamma(\lambda(f))=\Sigma_{12}\left(\int f(z)\left(\operatorname{id}_{L^{2}\left(G_{2}\right)} \otimes T_{z} \mathrm{~d} \mu_{1}(z)\right) \Sigma_{12}\right.
$$

in $\mathbb{B}\left(L^{2}\left(\widehat{G_{1}} \times G_{2} \times G_{1} \times G_{2}\right)\right)$. Finally, putting $s=1_{G_{2}}$ in (3.4) we obtain (3.3).

We gather some standard facts related to Fourier transform in the next lemma.

Lemma 3.5. Let $K$ be a compact subset of $G_{1}$. Define

$$
\begin{aligned}
& S(K, D):=\left\{F \in L^{1}\left(G_{1}, D\right) \mid \operatorname{supp}(F) \subset K\right\} \subset L^{1}(G, D) ; \\
& \widehat{S(K, D)}:=\{\widehat{F} \mid F \in S(K, D)\} \subset \mathrm{C}_{0}\left(\widehat{G_{1}}, D\right) .
\end{aligned}
$$

We have the following:

(1) Let $\widehat{G_{1}}$ is a Lie group, and let $\delta=\left(\delta_{1}, \cdots, \delta_{n}\right)$ be the canonical derivation on $\widehat{G_{1}}$ for $i=1, \cdots, n$. Then $\widehat{S(K, D)} \subset \operatorname{Dom}(\tilde{\delta})$ and $\tilde{\delta}(\widehat{S(K, D)}) \subset \widehat{S(K, D)}$, where $\tilde{\delta}_{i}:=\delta_{i} \otimes \operatorname{id}_{D}$ for $i=1, \cdots, n$, and $\tilde{\delta}:=\left(\tilde{\delta}_{1}, \cdots, \tilde{\delta}_{n}\right)$.

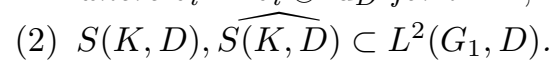

(3) Let $D^{\prime}$ be a $\mathrm{C}^{*}$-algebra and $\rho: D \rightarrow D^{\prime}$ be a completely bounded map. Then $(\mathrm{id} \otimes \rho) S(K, D) \subset S\left(K, D^{\prime}\right)$ and $(\mathrm{id} \otimes \rho) \widehat{S(K, D)} \subset \widehat{S\left(K, D^{\prime}\right)}$.

Proof. By definition $\delta_{i}\left(\lambda_{g}\right):=\rho_{i}(g) \lambda_{g}$, where $\lambda_{g} \in \mathcal{M}\left(\mathrm{C}_{\mathrm{r}}^{*}\left(G_{1}\right)\right) \cong \mathrm{C}_{\mathrm{b}}\left(\widehat{G_{1}}\right)$ and $\rho_{i} \in$ $\widehat{G_{1}}$ for $i=1, \cdots, n$; hence gives $(1)$. The second fact follows because the Fourier transform is $L^{2}$-isometry. The last fact is trivial.

For any $f \in \mathrm{C}_{\mathrm{c}}\left(G_{1}\right)$ and $\eta \in \mathrm{C}_{\mathrm{c}}\left(G_{2}\right)$ define $\hat{\pi}_{1}(f):=\lambda(f) \otimes 1$ and $\pi_{2}(\eta):=\alpha(\eta)$. Therefore, $\hat{\pi}_{1}(f) \pi_{2}(\eta) \in A$.

Proposition 3.6. Define $w:=\gamma\left(\hat{\pi}_{1}(f)\right)\left(1 \otimes \pi_{2}(\eta)\right) \in S(K, \mathcal{M}(A))$, where $K=$ $\operatorname{supp}(f)$. Then $p \mapsto(\operatorname{id} \otimes \varphi)\left(\hat{w}(p)^{*} \hat{w}(p)\right) \in \mathrm{C}_{0}\left(\widehat{G_{1}}\right)$.

Proof. By definition

$$
w^{*} w=\left(1 \otimes \pi_{2}\left(\eta^{*}\right)\right) \gamma\left(\hat{\pi}_{1}\left(|f|^{2}\right)\right)\left(1 \otimes \pi_{2}(\eta)\right)
$$

Using (2.15) we get

$$
\begin{aligned}
(\mathrm{id} \otimes \varphi)\left(w^{*} w\right) & =(\mathrm{id} \otimes \varphi)\left(1 \otimes \pi_{2}\left(\eta^{*}\right)\right) \gamma\left(\hat{\pi}_{1}\left(|f|^{2}\right)\right)\left(1 \otimes \pi_{2}(\eta)\right) \\
& =(\mathrm{id} \otimes \varphi)\left(1 \otimes \pi_{2}\left(|\eta|^{2}\right) \gamma\left(\hat{\pi}_{1}\left(|f|^{2}\right)\right)\right.
\end{aligned}
$$

By virtue of Proposition 3.2 and (2.15), for $p \in \widehat{G_{1}}$, we get

$$
\begin{aligned}
(\operatorname{id} \otimes \varphi)\left(\hat{w}(p)^{*} \hat{w}(p)\right) & =\iint|f|^{2}(g)|\eta|^{2}(s)\left\langle\beta_{s}(g), p\right\rangle \mathrm{d} \mu_{1}(g) \mathrm{d} \mu_{2}(s) \\
& =\iint|f|^{2}\left(\beta_{s^{-1}}\left(g^{\prime}\right)\right)|\eta|^{2}(s) \theta\left(g^{\prime}, s\right)\left\langle g^{\prime}, p\right\rangle \mathrm{d} \mu_{1}\left(g^{\prime}\right) \mathrm{d} \mu_{2}(s),
\end{aligned}
$$

where $g^{\prime}=\beta_{s}(g)$ and $\theta(g, s):=\left|\frac{\mathrm{d}}{\mathrm{d} \mu_{1}} \beta_{s^{-1}}(g)\right|$.

Define $G_{s}(g):=|f|^{2}\left(\beta_{s^{-1}}(g)\right)|\eta|^{2}(s) \theta(g, s)$. Then,

$$
(\mathrm{id} \otimes \varphi)\left(\hat{w}(p)^{*} \hat{w}(p)\right)=\int \widehat{G_{s}}(p) \mathrm{d} \mu_{2}(s) .
$$

A simple computation gives

$$
\begin{aligned}
\left\|G_{s}\right\|_{1}=\int\left\|G_{s}(g)\right\| \mathrm{d} \mu_{1}(g) & =\int|f|^{2}\left(\beta_{s^{-1}}(g)\right)|\eta|^{2}(s) \theta(g, s) \mathrm{d} \mu_{1}(g) \\
& =\int|f|^{2}(g)|\eta|^{2}(s) \mathrm{d} \mu_{1}(g)=\left(\|f\|_{2}\right)^{2}|\eta|^{2}(s) .
\end{aligned}
$$


Therefore, $\widehat{G_{s}} \in \mathrm{C}_{0}\left(\widehat{G_{1}}\right)$ for almost all $s \in G_{2}$. Also $\int|\eta|^{2}(s) \mathrm{d} \mu_{2}(s) \leq \infty$. By dominated convergence theorem, for any sequence $\left\{p_{n}\right\} \subset \widehat{G_{1}}$ such that $\left|p_{n}\right| \rightarrow \infty$ as $n \rightarrow \infty$, we have

$$
\lim _{n \rightarrow \infty} \int \widehat{G_{s}}\left(p_{n}\right) \mathrm{d} \mu_{2}(s)=\int\left(\lim _{n \rightarrow \infty} \widehat{G_{s}}\left(p_{n}\right)\right) \mathrm{d} \mu_{2}(s)=0 .
$$

Proof of Theorem 3.1. Proposition 3.2 and Proposition 3.6 give $\gamma$ is an element in $\operatorname{Mor}\left(\mathrm{C}_{0}\left(\widehat{G_{1}}\right), \mathrm{C}_{0}\left(\widehat{G_{1}}\right) \otimes A\right)$. The coassociativity of $\Delta_{A}$ gives (2.8) for $\gamma$.

Let $\left(L^{2}(\mathbb{G}), \pi, \Lambda\right)$ be the GNS triple for the the left Haar weight $\varphi$ in (2.15). For any $v \in L^{2}(\mathbb{G})$, define the operator $\Theta_{v}(c):=c v$ for $c \in \mathbb{C}$. Let $\left(e_{i}\right)_{i \in \mathbb{N}}$ be an orthonormal basis of $L^{2}(\mathbb{G})$. For $w \in S(K, \mathcal{M}(A))$ in Proposition 3.6, define

$$
x_{i}^{*}(p):=\left(\mathrm{id} \otimes \Theta_{e_{i}}^{*}\right)(\mathrm{id} \otimes \Lambda) \Delta_{A}(\hat{w}(p)) \in \mathcal{M}(A) \quad \text { for } p \in \widehat{G_{1}} .
$$

Also, for $q \in \mathcal{N}_{\varphi}$ define $q_{i}:=\left(\mathrm{id} \otimes \Theta_{e_{i}}^{*}\right)(\mathrm{id} \otimes \Lambda) \Delta_{A}(q) \in \mathcal{M}(A)$.

We compute,

$$
\begin{aligned}
\sum_{i=1}^{\infty} x_{i}(p) q_{i} & =\sum_{i=1}^{\infty}(\mathrm{id} \otimes \Lambda) \Delta_{A}(w(p))^{*}\left(1 \otimes \Theta_{e_{i}} \Theta_{e_{i}}^{*}\right)(\mathrm{id} \otimes \Lambda) \Delta_{A}(q) \\
& =(\operatorname{id} \otimes \Lambda) \Delta_{A}(w(p))^{*}(\mathrm{id} \otimes \Lambda) \Delta_{A}(q) \\
& =\varphi\left(\hat{w}(p)^{*} q\right) 1_{\mathcal{M}(A)}
\end{aligned}
$$

By virtue of Proposition 3.6, $F_{N}(p):=\Sigma_{i=1}^{N} x_{i}(p) q_{i}$ is strictly convergent. Hence, for any given $q^{\prime} \in A$, the sequence $\left\{F_{N}\left(1 \otimes q^{\prime}\right)\right\}$ converges uniformly over every compact subset of $\widehat{G_{1}}$.

In order to establish Podleś condition (2.9) for $\gamma$ we need to show $\left\{F_{N}\left(1 \otimes q^{\prime}\right)\right\}$ converges uniformly over $\widehat{G_{1}}$ for $q^{\prime} \in A$.

By a similar argument used in [16, Proposition 5.11], and Proposition [3.6 gives $\sum_{i=1}^{n} x_{i}^{*}(p) x_{i}(p)$ strictly converges to $\varphi\left(\hat{w}(p)^{*} \hat{w}(p)\right) 1_{\mathcal{M}(A)}$. Similarly, $\sum_{i=1}^{n} q_{i}^{*} q_{i}$ is bounded and strictly convergent. Let $\left\|\sum_{i=1}^{n} q_{i}^{*} q_{i}\right\|<C^{2}$. Given $\epsilon>0$, we can choose a compact subset $K^{\prime}$ of $\widehat{G_{1}}$, such that $(\mathrm{id} \otimes \varphi)\left(\hat{w}^{*}(p) \hat{w}(p)\right) \leq\left(\frac{\epsilon}{C}\right)^{2}$ for all $p \notin K^{\prime}$. Hence $\left\|\sum_{i=m}^{n} x_{i}(p) x_{i}^{*}(p)\right\| \leq\left(\frac{\epsilon}{C}\right)^{2}$ for all $p \notin K^{\prime}$, and for all $m, n$.

Now choose $N_{0}$ such that for all $m, n \geq N_{0},\left\|\left(F_{m}-F_{n}\right)(p) q^{\prime}\right\|<\epsilon$ for $p \in K^{\prime}$, $q^{\prime} \in A$.

Finally, for all $m, n \geq N_{0}$

$$
\left\|\left(F_{m}-F_{n}\right)(p)\right\| \leq\left\|\sum_{i=m}^{n} x_{i}(p) x_{i}^{*}(p)\right\|^{\frac{1}{2}}\left\|\sum_{i=m}^{n} q_{i}^{*} q_{i}\right\|^{\frac{1}{2}}<\epsilon\left\|q^{\prime}\right\|,
$$

for $p \notin K^{\prime}$. Hence $\left\{F_{N}\left(1 \otimes q^{\prime}\right)\right\}$ is Cauchy sequence in norm for $q^{\prime} \in A$.

\section{Properties of Bicrossed product $\mathrm{C}^{*}$-ACtions}

Let $G_{1}, G_{2}$, and $\mathbb{G}=\left(A, \Delta_{A}\right)$ be as before, so that $G_{1}$ is abelian. In this section we shall discuss various properties of the $\mathrm{C}^{*}$-action $\gamma$ of $\mathbb{G}$ on $\mathrm{C}_{0}\left(\widehat{G_{1}}\right)$ constructed in Theorem 3.1 .

Recall, a right action $\gamma$ of a von Neumann algebraic quantum group $\mathbb{G}=\left(M, \Delta_{M}\right)$ on a von Neumann algebra $N$ is called ergodic if $N^{\gamma}:=\left\{x \in N: \gamma(x)=x \otimes 1_{M}\right\}$ is equal to $\mathbb{C} \cdot 1_{N}$.

Proposition 4.1. The von Neumann algebraic action $\gamma$ of $\mathbb{G}$ on $L\left(G_{1}\right)$ is ergodic.

Proof. Let $\left(M, \Delta_{M}\right)$ be the von Neumann algebraic version of $\mathbb{G}$. By construction, $\gamma$ is obtained from the comultiplication $\Delta_{A}$ of the $\mathrm{C}^{*}$-algebraic version of $\mathbb{G}$. Since, $\Delta_{A}$ extends uniquely to the comultiplication $\Delta_{M}$ on $M$ by (2.14). Then, in a similar way, $\gamma$ also extends to a von Neumann algebraic action, denoted again by $\gamma$, of $\mathbb{G}$ 
on $L\left(G_{1}\right)$. Now ergodicity of $\Delta_{M}$ (see [10, Result 5.13] or [14, Theorem 2.1]) implies the same for $\gamma$.

4.1. Faithfulness. Motivated by [6, Definition 4.5] we propose the following definition, as a possible generalisation of faithful actions on locally compact quantum groups on $\mathrm{C}^{*}$-algebras.

Definition 4.2. A $\mathrm{C}^{*}$-action $\gamma: C \rightarrow C \otimes A$ of $\mathbb{G}=\left(A, \Delta_{A}\right)$ on a $\mathrm{C}^{*}$-algebra $C$ is called faithful if the ${ }^{*}$-algebra generated by $\left\{\left(\omega \otimes \operatorname{id}_{A}\right) \gamma(c): \omega \in C^{\prime}, c \in C\right\}$ is strictly dense in $\mathcal{M}(A)$.

Example 4.3. In particular, the comultiplication map $\Delta_{A}$ is a $\mathrm{C}^{*}$-action of $\mathbb{G}$ on $A$. Given any $\omega \in A^{\prime}$ and $a \in A$ define $\omega \cdot a \in A^{\prime}$ by $\omega \cdot a(b):=\omega(a b)$ for $b \in A$. Now the space $\left\{\omega \cdot a: \omega \in A^{\prime}, a \in A\right\}$ is weak *-dense in $A^{\prime}$. The cancellation property (2.1) of $\Delta_{A}$ shows that $\left\{\left(\omega \otimes \operatorname{id}_{A}\right) \Delta_{A}(A): \omega \in A\right.$ and $\left.a \in A\right\}$ is norm dense in $A$; hence $\Delta_{A}$ is a faithful $\mathrm{C}^{*}$-action.

Theorem 4.4. The $\mathrm{C}^{*}$-action $\gamma$ of $\mathbb{G}$ on $\mathrm{C}_{0}\left(\widehat{G_{1}}\right)$ is faithful if and only if $\beta$ is non-trivial.

Proof. If possible, assume that $\beta$ is trivial. Then Proposition 3.2 gives

$$
\gamma(\lambda(f))=\Delta_{\mathrm{C}_{\mathrm{r}}^{*}\left(G_{1}\right)}(\lambda(f)) \otimes 1 \quad \text { for all } f \in \mathrm{C}_{\mathrm{c}}\left(G_{1}\right) .
$$

Here $\Delta_{\mathrm{C}_{\mathrm{r}}^{*}\left(G_{1}\right)}$ denotes the comultiplication on $\mathrm{C}_{\mathrm{r}}^{*}\left(G_{1}\right)$. By Example 4.3 we observe that the set $\left\{\left(\omega \otimes \mathrm{id}_{A}\right) \gamma(\lambda(f)): \omega \in \mathrm{C}_{\mathrm{r}}^{*}\left(G_{1}\right)^{\prime}, f \in \mathrm{C}_{\mathrm{c}}\left(G_{1}\right)\right\}$ is strictly dense in $\mathcal{M}\left(\mathrm{C}_{\mathrm{r}}^{*}\left(G_{1}\right)\right.$; hence $\gamma$ is not faithful. Therefore, by contraposition we obtain that the faithfulness of $\gamma$ implies the nontriviality of $\beta$.

Conversely, assume that $\beta$ is non-trivial. By virtue of [1, Proposition 3.6] it is enough to show the set $\left\{\left(\omega \otimes \mathrm{id}_{A}\right) \gamma(\lambda(f)): \omega \in \mathrm{C}_{\mathrm{r}}^{*}\left(G_{1}\right)^{\prime}\right\}$ is norm dense in $\alpha\left(\mathrm{C}_{0}\left(G_{2}\right)\right) \cdot\left(\mathrm{C}_{\mathrm{r}}^{*}\left(G_{1}\right) \otimes 1\right)$.

Let $K$ and $K^{\prime}$ be compact subsets of $G_{1}$ such that $\mu_{1}(K) \neq 0$ and $\mu_{1}\left(K^{\prime}\right) \neq 0$. Let $\chi$ and $\chi^{\prime}$ be characteristic functions of $K$ and $K^{\prime}$ respectively. For a given $f \in$ $\mathrm{C}_{\mathrm{c}}\left(G_{1}\right)$ define

$$
A_{\chi, \chi^{\prime}}=\left(\omega_{\chi, \chi^{\prime}} \otimes \mathrm{id}_{A}\right) \gamma(\lambda(f))
$$

Here $\omega_{\chi, \chi^{\prime}}$ denotes the contraction with respect to $\chi_{K}$ and $\chi_{K^{\prime}}$.

For all $\xi \in L^{2}\left(G_{1} \times G_{2}\right)$ we get,

$$
\begin{aligned}
A_{\chi, \chi^{\prime}} \xi(h, t) & =\int \overline{\chi(g)} \gamma(\lambda(f))\left(\chi^{\prime}(g) \otimes \xi(h, t) \mathrm{d} \mu_{1}(g)\right. \\
& =\iint \overline{\chi(g)} f(z) \chi^{\prime}\left(g \beta_{\alpha_{h}(t)}(z)\right) \xi(h z, t) \mathrm{d} \mu_{1}(g) \mathrm{d} \mu_{1}(z) \\
& =\int \mu_{1}\left(K \cap \beta_{\alpha_{h}(t)}(z) K^{\prime}\right) f(z) \xi(h z, t) \mathrm{d} \mu_{1}(z)
\end{aligned}
$$

Let $\left\{K_{n}\right\}_{n \in \mathbb{N}}$ be an increasing sequence of compact subsets of $G_{1}$ and growing up to the whole group $G_{1}$. Let $\chi_{n}$ denotes the characteristic function of $K_{n}$ for all $n \in \mathbb{N}$. By dominated convergence theorem,

$$
\lim _{n \rightarrow \infty} A_{\chi_{n}, \chi^{\prime}} \xi(h, t)=\int \mu_{1}\left(\beta_{\alpha_{h}(t)}(z) K^{\prime}\right) f(z) \xi(h z, t) \mathrm{d} \mu_{1}(z) .
$$

Invariance of Haar measure $\mu_{1}$ gives $\mu_{1}\left(\beta_{\alpha_{h}(t)}(z) K^{\prime}\right)=\mu_{1}\left(K^{\prime}\right)$ for all $h, z \in G_{1}$, $t \in G_{2}$. Therefore,

$$
\lim _{n \rightarrow \infty} A_{\chi_{n}, \chi^{\prime}} \xi(h, t)=\mu_{1}\left(K^{\prime}\right) \int f(z) \xi(h z, t) \mathrm{d} \mu_{1}(z)=\mu_{1}\left(K^{\prime}\right)(\lambda(f) \otimes 1) \xi(h, t) .
$$

Thus $A_{\chi_{n}, \chi}$ goes strictly to $\lambda_{g_{0}}$ for some $g_{0} \in G_{1}$. Therefore, for any $\eta, \eta^{\prime} \in L^{2}\left(G_{1}\right)$, the operator $B_{\eta, \eta^{\prime}, g_{0}}:=\left(\omega_{\eta, \eta^{\prime}} \otimes \operatorname{id}_{A}\right) \gamma\left(\lambda\left(g_{0}\right)\right)\left(\lambda_{g_{0}^{-1}} \otimes 1\right)$ is in the desired algebra. 
Next we compute,

$$
\begin{aligned}
B_{\eta, \eta^{\prime}, g_{0}} \xi(h, t) & =\left(\left(\omega_{\eta, \eta^{\prime}} \otimes \operatorname{id}_{A}\right) \gamma\left(\lambda\left(g_{0}\right)\right)\right)\left(\lambda_{g_{0}^{-1}} \otimes 1\right) \xi(h, t) \\
& =\left(\lambda_{g_{0}^{-1}} \otimes 1\right) \int \overline{\eta(g)} \eta^{\prime}\left(g \beta_{\alpha_{h}(t)}\left(g_{0}\right)\right) \xi\left(h g_{0}, t\right) \mathrm{d} \mu_{1}(g) \\
& =\int \overline{\eta(g)} \eta^{\prime}\left(g \beta_{\alpha_{h}(t)}\left(g_{0}\right)\right) \mathrm{d} \mu_{1}(g) \xi(h, t), \quad \text { for } \xi \in L^{2}\left(G_{1} \times G_{2}\right) .
\end{aligned}
$$

Since, $\beta$ is non-trivial, varying $\eta, \eta^{\prime}, g_{0}$ and using Stone-Weierstrass theorem we get the set of functions $x \rightarrow \int \overline{\eta(g)} \eta^{\prime}\left(g \beta_{x}\left(g_{0}\right)\right) \mathrm{d} \mu_{1}(g)$ for $x \in G_{2}$ which is norm dense in $\mathrm{C}_{0}\left(G_{2}\right)$. Therefore, the set of operators $B_{\eta, \eta^{\prime}, g_{0}}$ is norm dense in $\alpha\left(\mathrm{C}_{0}\left(G_{2}\right)\right)$.

4.2. Isometry. We recall the definition of isometric action from [5] for compact quantum groups. In analogy to this, it is natural to make the following definition of isometric action in the locally compact set-up:

Definition 4.5. Let $\gamma$ be a $\mathrm{C}^{*}$-action of a locally compact quantum group $\mathbb{G}$ on $\mathrm{C}_{0}(X)$ where $X$ is a smooth Riemannian (possibly non-compact) manifold with the Hodge-Laplacian $\mathcal{L}=-d^{*} d$, where $d$ denotes the de-Rham differential operator. We say that $\gamma$ is for every bounded linear functional $\omega$ on $M(Q)$, (id $\otimes \omega) \circ \gamma$ maps $\mathrm{C}_{\mathrm{b}}^{\infty}(X)$ to itself and commutes with $\mathcal{L}$ on that subspace.

Just as in [6], it is easy to prove that for any isometric action $\gamma$ and any smooth vector field $\chi$ on $X, f, \phi \in \mathrm{C}_{\mathrm{b}}^{\infty}(X),(\chi \otimes \mathrm{id})(\gamma(f))$ and $\gamma(\phi)$ will commute.

Proposition 4.6. Assume $\widehat{G_{1}}$ is a Lie group. Then the $\mathrm{C}^{*}$-action $\gamma$ of $\mathbb{G}$ on $\mathrm{C}_{0}\left(\widehat{G_{1}}\right)$ is isometric whenever either $\alpha$ or $\beta$ is trivial.

Proof. As noted before, the condition of isometry of $\gamma$ implies the operators $\left(\delta_{i} \otimes\right.$ $\left.\operatorname{id}_{A}\right) \gamma\left(\lambda_{g_{1}}\right)$ and $\gamma\left(\lambda_{g_{2}}\right)$ commute for all $g_{1}, g_{2} \in G_{1}$ and derivations $\delta_{i}$ on $\widehat{G_{1}}$.

Let $\xi \in L^{2}\left(G_{1} \times G_{1} \times G_{2}\right)$. Using Proposition 3.2, we compute

$$
\begin{aligned}
L & =\left(\delta_{i} \otimes \operatorname{id}_{A}\right) \gamma\left(\lambda_{g_{1}}\right) \gamma\left(\lambda_{g_{2}}\right) \xi(g, h, t) \\
& =\rho_{i}\left(\beta_{\alpha_{h}(t)}\left(g_{1}\right)\right) \gamma\left(\lambda_{g_{2}}\right) \xi\left(g \beta_{\alpha_{h}(t)}\left(g_{1}\right), h g_{1}, t\right) \\
& =\rho_{i}\left(\beta_{\alpha_{h}(t)}\left(g_{1}\right)\right) \xi\left(g \beta_{\alpha_{h}(t)}\left(g_{1}\right) \beta_{\alpha_{h g_{1}}(t)}\left(g_{2}\right), h g_{1} g_{2}, t\right)
\end{aligned}
$$

Using (2.13) and commutativity of $G_{1}$ we get,

$$
L=\rho_{i}\left(\beta_{\alpha_{h}(t)}\left(g_{1}\right)\right) \xi\left(g \beta_{\alpha_{h}(t)}\left(g_{1} g_{2}\right), h g_{1} g_{2}, t\right) .
$$

A similar computation gives

$$
R=\gamma\left(\lambda_{g_{2}}\right)\left(\delta_{i} \otimes \operatorname{id}_{A}\right) \gamma\left(\lambda_{g_{1}}\right) \xi(g, h, t)=\rho_{i}\left(\beta_{\alpha_{h g_{2}}(t)}\left(g_{1}\right)\right) \xi\left(g \beta_{\alpha_{h}(t)}\left(g_{1} g_{2}\right), h g_{1} g_{2}, t\right) .
$$

Now $L=R$ for all $\xi \in L^{2}\left(G_{1} \times G_{1} \times G_{2}\right), g_{1}, g_{2} \in G_{1}$, and $\rho_{i} \in \widehat{G_{1}}$, implies

$$
\beta_{\alpha_{h}(t)}\left(g_{1}\right)=\beta_{\alpha_{h g_{2}}(t)}\left(g_{1}\right) \quad \text { for all } g_{1}, g_{2}, h \in G_{1}, t \in G_{2} .
$$

This is true if either of the actions $\alpha$ or $\beta$ is trivial.

We prove the main result of this section:

Theorem 4.7. Assume $\widehat{G_{1}}$ is a Lie group and the $\mathrm{C}^{*}$-action $\gamma$ of $\mathbb{G}$ on $\mathrm{C}_{0}\left(\widehat{G_{1}}\right)$ is faithful and isometric. Then $\mathbb{G}$ is classical group.

Proof. By Theorem 4.4 faithfulness of $\gamma$ implies that $\beta$ is non-trivial. On the other hand, $\gamma$ is isometric; hence Proposition 4.6 forces $\alpha$ to be trivial. From (2.14) and using the fact that $G_{1}$ is abelian we get $M=L^{\infty}\left(\widehat{G_{1}} \times G_{2}\right)$. 


\section{EXAMPLES}

' $a x+b$ ' is the group of affine transformations of the real line $\mathbb{R}$. The natural action of $a x+b$ on $\mathbb{R}$ given by $x \mapsto a x+b$ for $a \in \mathbb{R} \backslash\{0\}$ and $b, x \in \mathbb{R}$ is faithful. We apply our results on two versions of quantum $a x+b$ group discussed in [15, Section 5]. Both of them are genuine non-compact, non-discrete, non-Kac quantum groups. We show that they act ergodically and faithfully on non-compact Riemannian manifolds. However none of these actions are not isometric.

5.1. Baaj-Skandalis' ax $+\mathbf{b}$ group. Assume $G_{1}=G_{2}=\mathbb{R} \backslash\{0\}$ and the group operation is the usual multiplication. Let $G=\{(a, b) \mid a \in \mathbb{R} \backslash\{0\}, b \in \mathbb{R}\}$ with $(a, b)(c, d)=(a c, d+c b)$.

Define $i: G_{1} \mapsto G$ and $j: G_{2} \mapsto G$ by

$$
i(g):=(g, g-1), \quad j(s):=(s, 0),
$$

for all $g \in G_{1}$ and $s \in G_{2}$. This way $\left(G_{1}, G_{2}\right)$ is a matched pair in the sense of Definition 2.11. Associated actions $\alpha$ and $\beta$ are defined by

$$
\alpha_{g}(s)=\frac{g s}{s(g-1)+1}, \quad \beta_{s}(g):=s(g-1)+1 .
$$

for all $g, s \in \mathbb{R} \backslash\{0\}$ such that $(g-1) \neq-s^{-1}$.

Associated bicrossed product $\mathbb{G}$ is the Baaj-Skandalis' quantum $a x+b$ group (see [15, Section 5.3]). By [15, Proposition 5.2 \& 5.3], $\mathbb{G}$ is self dual, non-Kac, non-compact, non-discrete quantum group.

Proposition 5.1. There is an ergodic, faithful and non-isometric $\mathrm{C}^{*}$-action of $\mathbb{G}$ on $\mathrm{C}_{0}(\mathbb{R} \backslash\{0\})$.

Proof. Clearly, $G_{1}$ is abelian and $\widehat{G_{1}}$ is a Lie group with two connected components. Since $\beta$ is non-trivial and $\mathbb{G}$ is a genuine quantum group, by Proposition 4.1 Theorem 4.4, and Theorem 4.7 the $C^{*}$-action $\gamma$ of $\mathbb{G}$ on $\mathrm{C}_{0}(\mathbb{R} \backslash\{0\})$ in Theorem 3.1 is ergodic, faithful and not isometric.

5.2. Split-Extension. Assume $G_{1}=\{(a, b) \mid a>0, b \in \mathbb{R}\}$ with $(a, b)(c, d):=$ $\left(a c, a d+\frac{b}{c}\right)$ and $G_{2}=(\mathbb{R},+)$. Let $K$ be the multiplicative group with two elements. Define $G=S L_{2}(\mathbb{R}) / K$, and $i: G_{1} \mapsto G, j: G_{2} \mapsto G$ by

$$
i(a, b):=\left(\begin{array}{cc}
a & b \\
0 & \frac{1}{a}
\end{array}\right) \bmod K, \quad j(x):=\left(\begin{array}{cc}
1 & 0 \\
x & 1
\end{array}\right) \bmod K .
$$

This way $\left(G_{1}, G_{2}\right)$ is a matched pair in the sense of Definition 2.11. Associated actions $\alpha$ and $\beta$ are defined by

$$
\alpha_{(a, b)}(x):=\frac{x}{a(a+b x)}, \quad \beta_{x}(a, b):= \begin{cases}(a+b x, b) & \text { if } a+b x>0, \\ (-a-b x,-b) & \text { if } a+b x<0 .\end{cases}
$$

whenever $a x+b \neq 0$.

By [15, Proposition 5.5], associated bicrossed product $\mathbb{G}$ and its dual $\widehat{\mathbb{G}}$ are non-Kac, non-compact, and non-discrete quantum group. Also, $\widehat{\mathbb{G}}$ is not unimodular. Moreover, [15, Remark 5.6] shows that $\widehat{\mathbb{G}}$ is deformation of some generalised $a x+b$ group.

Proposition 5.2. There is an ergodic, faithful and non-isometric $\mathrm{C}^{*}$-action of $\widehat{\mathbb{G}}$ on $\mathrm{C}_{0}(\mathbb{R})$.

Proof. Clearly, $G_{2}$ is an abelian and $\widehat{G_{2}}$ is a connected Lie group. Recall that $\widehat{\mathbb{G}}$ is obtained by exchanging $G_{1}$ and $\alpha$ with $G_{2}$ and $\beta$. Since $\alpha$ is non-trivial, Proposition 4.1. Theorem 4.4, and Theorem 4.7 give the $\mathrm{C}^{*}$-action $\gamma$ of $\widehat{\mathbb{G}}$ on $\mathrm{C}_{0}(\mathbb{R})$ in Theorem 3.1 is ergodic, faithful and not isometric. 


\section{REFERENCES}

[1] Saad Baaj, Georges Skandalis, and Stefaan Vaes, Non-semi-regular quantum groups coming from number theory, Comm. Math. Phys. 235 (2003), no. 1, 139-167, doi: $10.1007 /$ s00220-002-0780-6 MR 1969723

[2] Saad Baaj and Stefaan Vaes, Double crossed products of locally compact quantum groups, J. Inst. Math. Jussieu 4 (2005), no. 1, 135-173, doi: 10.1017/S1474748005000034 MR 2115071

[3] Vladimir Gershonovich Drinfel'd, Quantum groups, Proceedings of the International Congress of Mathematicians, Vol. 1, 2 (Berkeley, Calif., 1986), 1987, pp. 798-820. MR 934283

[4] Pavel Etingof and Chelsea Walton, Semisimple Hopf actions on commutative domains, Adv. Math. 251 (2014), 47-61, doi: 10.1016/j.aim.2013.10.008 MR 3130334

[5] Debashish Goswami, Quantum group of isometries in classical and noncommutative geometry, Comm. Math. Phys. 285 (2009), no. 1, 141-160, doi: 10.1007/s00220-008-046 MAR, 2453592

[6] Debashish Goswami and Soumalya Joardar, Rigidity of action of compact quantum groups on compact, connected manifolds, eprint. arXiv:1309.1294

[7] Huichi Huang, Faithful compact quantum group actions on connected compact metrizable spaces, J. Geom. Phys. 70 (2013), 232-236, doi: 10.1016/j.geomphys.2013.03.027MR 3054297

[8] Invariant subsets under compact quantum group actions (2013), eprint. arXiv: $1210.5782 \mathrm{v} 2$

[9] Michio Jimbo, A q-difference analogue of $U(\mathfrak{g})$ and the Yang-Baxter equation, Lett. Math. Phys. 10 (1985), no. 1, 63-69, doi: 10.1007/BF00704588 MR 797001

[10] Johan Kustermans and Stefaan Vaes, Locally compact quantum groups, Ann. Sci. École Norm. Sup. (4) 33 (2000), no. 6, 837-934, doi: 10.1016/S0012-9593(00)01055-7 |MR 1832993

[11] Locally compact quantum groups in the von Neumann algebraic setting, Math. Scand. 92 (2003), no. 1, 68-92, available at http://www.mscand.dk/article.php?id=19\$IR 1951446

[12] Yuri Ivanovich Manin, Quantum groups and noncommutative geometry, Université de Montréal, Centre de Recherches Mathématiques, Montreal, QC, 1988. MR 1016381

[13] Tetsuya Masuda, Yoshiomi Nakagami, and Stanisław Lech Woronowicz, A $C^{*}$-algebraic framework for quantum groups, Internat. J. Math 14 (2003), no. 9, 903-1001, doi: 10.1142/S0129167X03002071 MR 2020804

[14] Ralf Meyer, Sutanu Roy, and Stanisław Lech Woronowicz, Homomorphisms of quantum groups, Münster J. Math. 5 (2012), 1-24, available at http://nbn-resolving.de/urn:nbn:de:hbz:6-88399662599, MR 3047623

[15] Stefaan Vaes and Leonid Vainerman, Extensions of locally compact quantum groups and the bicrossed product construction, Adv. Math. 175 (2003), no. 1, 1-101, doi: 10.1016/S0001-8708(02)00040-3 MR 1970242

[16] Stefaan Vaes and Alfons Van Daele, Hopf $C^{*}$-algebras, Proc. London Math. Soc. (3) 82 (2001), no. 2, 337-384, doi: 10.1112/S002461150101276X MR 1806875

[17] Stanisław Lech Woronowicz, Compact quantum groups, Symétries quantiques (Les Houches, 1995), North-Holland, Amsterdam, 1998, pp. 845-884. MR 1616348

[18] _ From multiplicative unitaries to quantum groups, Internat. J. Math. 7 (1996), no. 1, 127-149, doi: 10.1142/S0129167X96000086 MR 1369908

E-mail address: goswamid@isical.ac.in

Statistics and Mathematics Unit, Indian Statistical Institute, 203, B. T. Road, Kolkata 700108, INDIA

E-mail address: sutanu.roy@carleton.ca

School of Mathematics and Statistics, Carleton University, 1125 Colonel By Drive, Ottawa, ON K1S 5B6, Canada 